\title{
Synthesis of nanostructured vanadium phosphate catalysts using sonochemical route for partial oxidation of $\mathbf{n}$-butane.
}

\begin{abstract}
In this study, sonochemical treatment is used to synthesize nanostructured vanadium pentoxide, V2O5 with different duration of time andmineralizers. Eight samples of V2O5 have been prepared using $\mathrm{KN} 03$ and $\mathrm{KCl}$ as mineralizers that undergo sonochemical treatment with different duration i.e., 30, 60, 90 and $120 \mathrm{~min}$, respectively. These samples were denoted as KNO30, KNO60, KNO90, KNO120, KC130, KC160, KC190 and KC1120. Nanostructured V2O5 prepared via ultrasound irradiation for $30 \mathrm{~min}$ in $\mathrm{KC} 1$ (KC130) was chosen as starting material to synthesis vanadium phosphorus oxide catalyst (denoted VPOS30KC1). All the materials synthesized was characterized by using X-Ray Diffraction (XRD), Brunauer-Emmett-Teller surface area measurement (BET), chemical analysis, Transmission Electron Microscopy (TEM), Scanning Electron Microscopy (SEM) and temperature programmed reduction (H2-TPR). Catalytic properties for n-butane oxidation of the catalyst, VPOS30KC1 was investigated by using a fixed-bed microreactor at $673 \mathrm{~K}$ (GHSV $=2400 \mathrm{~h} 1)$. SEM micrographs show that the morphology of the V2O5 changed from platelet-like particles intonanorods after $90 \mathrm{~min}$ of sonochemical treatment. XRD patterns detected that the potassium ion from the mineralizers was incorporated into the V2O5 structure after prolonged sonochemical treatment duration. $\mathrm{H}$ j-TPR profiles shown that VPOS30KC1 gave higher amount of reactive oxygen species $(0-\mathrm{V} 4+)$ removed which will expect to give higher activity. Catalytic evaluation showed that VPOS30KC1 gave higher conversion (19\%) while retaining the selectivity towards maleic anhydride (48\%) compared to the bulk VPO catalyst prepared in the same organic medium (XC4: 14\%).
\end{abstract}

Keyword: Sonochemical treatment; Ultrasound irradiation; Vanadium phosphorus oxide; Butane oxidation. 